\title{
Investigations into Some Simple Expressions of the Gamma Function in Wind Power Theoretical Estimate by the Weibull Distribution
}

\author{
Siaka Touré \\ Solar Energy Laboratory, FHB University Abidjan Cocody, Abidjan, Côte d'Ivoire \\ Email: siakaahtoure@yahoo.fr
}

How to cite this paper: Touré, S. (2019) Investigations into Some Simple Expressions of the Gamma Function in Wind Power Theoretical Estimate by the Weibull Distribution. Journal of Applied Mathematics and Physics, 7, 2990-3002. https://doi.org/10.4236/jamp.2019.712209

Received: September 27, 2019

Accepted: December 6, 2019

Published: December 9, 2019

Copyright $\odot 2019$ by author(s) and Scientific Research Publishing Inc. This work is licensed under the Creative Commons Attribution International License (CC BY 4.0).

http://creativecommons.org/licenses/by/4.0/

\begin{abstract}
The Weibull distribution is a probability density function (PDF) which is widely used in the study of meteorological data. The statistical analysis of the wind speed $v$ by using the Weibull distribution leads to the estimate of the mean wind speed $\langle v\rangle$, the variance of $v$ around $\langle v\rangle$ and the mean power density in the wind. The gamma function $\Gamma$ is involved in those calculations, particularly $\Gamma(1+1 / k), \Gamma(1+2 / k)$ and $\Gamma(1+3 / k)$. The paper reports the use of the Weibull PDF $f(v)$ to estimate the gamma function. The study was performed by looking for the wind speeds related to the maximum values of $f$ (v), $v^{2} f(v)$ and $v^{3} f(v)$. As a result, some approximate relationships were obtained for $\Gamma(1+1 / k), \Gamma(1+2 / k)$ and $\Gamma(1+3 / k)$, that use some fitting polynomial functions. Very good agreements were found between the exact and the estimated values of $\Gamma(1+n / k)$ that can be used for the estimation of the mean wind speed $\langle v\rangle$, the variance $\sigma^{2}$ of the wind speed $v$ around the mean speed and the average wind power density.
\end{abstract}

\section{Keywords}

Gamma Function, Weibull Distribution, Beta Function, Mean Wind Speed, Average Wind Power Density

\section{Introduction}

A growing interest in renewable sources of energy is observed, owing to the increase in electricity demand and the world environmental concern. Due to its cleanness, wind energy becomes more and more attractive. A Wind Energy 
Conversion System (WECS) produces electricity from wind power. The production price depends on several factors. One of those factors is the amount of wind available at a site. Therefore, a reliable estimate of wind energy potential at a selected site is important. For wind energy applications and more generally for weather forecast, wind speed is analysed. Samples are set out from real recordings of wind speed. Then the fitting parameters are looked for to find the distribution that fits the best the data recorded. Several probability density functions (PDF) are used in the study of meteorological data, such as the 2-parameter Weibull distribution, the 3-parameter Weibull distribution or the mixture Weibull distribution which are widely used in wind energy potential analysis. This Weibull model is often used for the assessments of the mean wind speed and the mean power density. It is worth noting that the gamma function is involved in those assessments. The Flexible Weibull Extension is also used to study the distribution of random variables [1]. The beta PDF is also used in the study of meteorological data. All those PDF calculations use the gamma function. This gamma function is also very important because it is involved in several Laplace transform calculations. The Laplace transform is a tool for solving differential equations. Let a function $f(t)$ be considered. Its Laplace transform is defined as [2]:

$$
L[f(t)](s)=\int_{0}^{\infty} f(t) \mathrm{e}^{-s t} \mathrm{~d} t .
$$

For instance, if $f(t)=t^{x}$, one gets, for a real number $x>-1$

$$
L\left[t^{x}\right](s)=\frac{\Gamma(x+1)}{s^{x+1}} .
$$

In Equation (2), $\Gamma$ is the gamma function. So far, some tables exist, that give approximate values of the gamma function. The objective of the present work is to find some simple expressions of the gamma function, linked to a fitting parameter of the Weibull PDF, which may be used in wind power estimate and in the study of other meteorological data.

\section{The Gamma Function and Some Related Special Functions}

The gamma function was first introduced by Leonhard Euler in 1729 and 1730 [3] [4] [5] [6] Euler defined this function as follows:

For $x>0$,

$$
\Gamma(x)=\int_{0}^{1}(-\log (t))^{x-1} \mathrm{~d} t .
$$

When the following change of variable $u=-\log (t)$ is performed, the gamma function becomes:

$$
\Gamma(x)=\int_{0}^{\infty} t^{x-1} \exp (-t) \mathrm{d} t .
$$

For $x>0$, the above relationship can be written as:

$$
\Gamma(x+1)=\int_{0}^{\infty} t^{x} \exp (-t) \mathrm{d} t .
$$

From Equation (5), integration by parts yields the following recursion formula, commonly known as the functional equation: 


$$
\Gamma(x+1)=x \Gamma(x) .
$$

When $x$ is an integer number, the functional equation becomes:

$$
\Gamma(n+1)=n ! .
$$

Hence, the gamma function can be considered as a generalization of the factorial function to real non null positive numbers.

The gamma function is often approximated by infinite products or infinite series. For instance, the Weierstrass definition defines the gamma function as an infinite product [7]:

$$
\frac{1}{\Gamma(x)}=x \mathrm{e}^{\gamma x} \prod_{p=1}^{\infty}\left(1+\frac{x}{p}\right) \mathrm{e}^{-x / p}
$$

$X$ is any real number which should not be a negative integer $(0,-1,-2, \cdots), \gamma$ is the Euler's constant.

For large integer values $n$, the Stirling formula is used. It is expressed as:

$$
\Gamma(n+1)=n ! \sim \sqrt{2 \pi n} n^{n} \mathrm{e}^{-n} .
$$

For $x \rightarrow \infty$, the Stirling asymptotic formula is expressed as [8]:

$$
\Gamma(x+1)=\sqrt{2 \pi x} x \mathrm{e}^{-x}\left(1+\frac{1}{12 x}+\frac{1}{288 x^{2}}-\frac{139}{51840 x^{3}}-\frac{571}{2488320 x^{4}}+\cdots\right) .
$$

The eulerian beta function $B(x, y), x>0, y>0$ is related to the gamma function. It is expressed as:

$$
B(x, y)=\int_{0}^{1} t^{x-1}(1-t)^{y-1} \mathrm{~d} t .
$$

The gamma function and the beta function are related by the following equation:

$$
B(x, y)=\frac{\Gamma(x) \Gamma(y)}{\Gamma(x+y)} .
$$

The beta function is often used in the statistical analysis of sunshine duration data and in other meteorological data, such as the wind speed [9] [10] [11] [12].

In those studies, the probability distribution function of a random variable $x$ which ranges from 0 to 1 , may be expressed as a beta kind and expressed as:

$$
f(x)=x^{p-1}(1-x)^{q-1} / B(p, q) .
$$

$p$ and $q$ are the fitting parameters of the beta PDF.

The revue made above shows that, so far, no simple expressions of the gamma function are known. As a matter of fact, the values of the gamma function are found in tables, where the arguments lie on the interval [1] [2] with an increment of 0.001 [7].

\section{The 2-Parameter Weibull Distribution and the Gamma Function}

For a given wind speed $v$, the 2-parameter Weibull distribution is expressed by the following probability density function (PDF) [13]: 


$$
f(v)=\left(\frac{k}{c}\right)(v / c)^{k-1} \exp \left[-(v / c)^{k}\right], k>0, c>0 .
$$

$k$ is the dimensionless shape parameter whereas $c\left(\mathrm{~m} \cdot \mathrm{s}^{-1}\right)$ is the scale parameter. This Weibull distribution has been used by several authors. For instance, Manish Kumar and Cherian Samuel [14] considered in their study the Weibull, Gamma and Lognormal probability distributions to look for the best-fitted distribution. The result showed that, for the wind speed data of their selected site, the Weibull distribution is the best-fitted distribution.

These fitting parameters $k$ and $c$ are determined by using several procedures such as the regression method [15] [16] [17], the maximum likelihood procedure [18] [19] and the Eigen-coordinates method [20]. The nth moment of the Weibull random variable $v$ is expressed as:

$$
E\left(v^{n}\right)=\int_{0}^{\infty} v^{n} f(v) \mathrm{d} v=c^{n} \Gamma\left(1+\frac{n}{k}\right) .
$$

Then the first moment (which is the mean speed) $\langle v\rangle$ and the second moment $\left\langle v^{2}\right\rangle$ are expressed as:

$$
\begin{gathered}
\langle v\rangle=c \Gamma\left(1+\frac{1}{k}\right) \\
\left\langle v^{2}\right\rangle=c^{2} \Gamma\left(1+\frac{2}{k}\right) .
\end{gathered}
$$

Hence $\langle v\rangle$ and $\left\langle v^{2}\right\rangle$ are functions of $k$ and $c$, as well as the variance $\sigma^{2}$ of the wind speed $v$ around the mean speed, which is given by $\sigma^{2}=\left\langle v^{2}\right\rangle-\langle v\rangle^{2}$. On another hand, the theoretical wind power density is expressed as $P=1 / 2 \rho v^{3}$, where $\rho$ is the density of air. Then the average wind power density is given by:

$$
\langle P\rangle=\frac{1}{2} \rho c^{3} \Gamma\left(1+\frac{3}{k}\right) .
$$

Equation (15) shows that the Weibull distribution $f(v)$ may be used to estimate $\Gamma(1+n / k)$ which is involved in the wind energy potential assessment.

\section{Some Simple Expressions of the Gamma Function}

\subsection{Some Previous Works Related to the Subject}

Some previous works establish a direct link between $\langle v\rangle$ and $k$. An example is the formula given by Justus in 1978 for 140 sites in the USA [21] It is expressed as:

$$
k=d_{1} \sqrt{\langle v\rangle} .
$$

$k$ is the Weibull shape parameter; the constant $d_{1}$ is site specific. Equation (19) is a relation between the mean speed $\langle v\rangle$ and the shape parameter $k$.

Another interesting relationship is the one given by Gash and Twele. It is expressed as [22]:

$$
\langle v\rangle=c\left(0.287 k^{-1}+0.688\right)^{1 / k} .
$$


This relationship is interesting because, like Equation (16), it expresses the mean speed $\langle v\rangle$ as a function of the Weibull fitting parameters $k$ and $c$.

Hence, the reliability of Equation (20) was tested by using the data that we reported in our previous study of wind power potential in Côte d'Ivoire (TOURE and LAOUALY, 2000). An estimated value $\left\langle v_{c}\right\rangle$ of the mean wind speed was calculated by using Equation (20) with the Weibull parameters $k$ and $c$ found for Korhogo, a city located in the north of Côte d'Ivoire. Table 1 displays not only $\left\langle v_{c}\right\rangle$, but also the experimental mean wind speed $\left\langle v_{e x}\right\rangle$ and the relative error between $\left\langle v_{c}\right\rangle$ and $\left\langle v_{e x}\right\rangle$.

A fairly good agreement is observed between experimental and calculated values of $\langle v\rangle$.

So the Equation (20) given by Gash and Twele gives a good estimate of the mean speed $\langle v\rangle$. It should be noted that Equations (16) and (20) are both expressions of $\langle v\rangle$. A comparison between the two equations shows that $\Gamma(1+1 / k)$ can be expressed as:

$$
\Gamma(1+1 / k)=\left(0.287 k^{-1}+0.688\right)^{1 / k} .
$$

Equation (21) can be transformed as follows:

$$
\Gamma\left(1+\frac{1}{k}\right)=\left[0.688\left(1+\frac{0.287 / 0.688}{k}\right)\right]^{1 / k} .
$$

Then one gets

$$
\Gamma\left(1+\frac{1}{k}\right)=0.688^{\frac{1}{k}}\left(1+\frac{b}{k}\right)^{1 / k} .
$$

In Equation (23), $b=0.287 / 0.688$.

Moreover, a fitting number $a$ is introduced, by expressing $0.688^{1 / k}$ as:

$$
0.688^{1 / k}=\frac{0.688}{k} a .
$$

As a matter of fact, the fitting number depends on $k$. Hence $a(k)$ was defined as:

$$
a(k)=0.688 a .
$$

Finally, the transformation of Equation (21) leads to the following relationship:

$$
\Gamma\left(1+\frac{1}{k}\right)=\frac{a(k)}{k}\left(1+\frac{b}{k}\right)^{1 / k} .
$$

Then investigations were made to find how Equation (25) can be used as a basis to find some simple expressions of $\Gamma(1+n / k)$.

\subsection{Some Simple Expressions of $\Gamma(1+1 / k), \Gamma(1+2 / k)$ and}

\section{$\Gamma(1+2 / k)$ in Wind Power Theoretical Estimate}

\subsection{1. $\Gamma(1+1 / k)$ Estimate}

The Weibull distribution $f(v)$, given by Equation (14), was used to find an esti- 
mate of $\Gamma(1+1 / k)$. The value of the wind speed $v$ for which $f(v)$ is maximum, $V_{m p}$, is a solution of the equation

$$
\mathrm{d} f(v) / \mathrm{d} v=0 .
$$

It is easily found that

$$
v_{m p}=c(1-1 / k)^{1 / k} .
$$

$V_{m p}$ and the mean wind speed $\langle v\rangle$ given by Equation (16) are correlated. For instance, in the case of the Rayleigh distribution, for which $k=2$, one gets

$$
v_{m p}=\frac{2}{\sqrt{2 \pi}}\langle v\rangle .
$$

Therefore, Equations (16) and (27) suggest a link between $\Gamma(1+1 / k)$ and $(1-1 / k)^{1 / k}$. This link was established by using Equation (25), so that one gets:

$$
\Gamma\left(1+\frac{1}{k}\right)=\frac{a_{1}(k)}{k}\left(1-\frac{1}{k}\right)^{1 / k} .
$$

A comparison between Equations (25) and (28) shows that, $b=b(1)=-1$. As $\Gamma(1+1 / k)$ is the value of $\Gamma(1+n / k)$ for $n=1, a_{1}(k)$ is the fitting number $a(k)$ for $n=1 . b(1)=b=-1$ is the value of $b(n)$ for $n=1$. The fitting number $a_{1}(k)$ was investigated.

For each value of $k$, the exact value of $\Gamma(1+1 / k)$ was calculated by using tables. Then by using Equation (28), $a_{1}(k)$ was found. Subsequently, $a_{1}(k)$ was plotted against $k$.

This plotting is given in Figure 1. It was found that $a_{1}(k)$ is a polynomial function of $k$. The following relationship was found for $k \leq 8$, with $R^{2}=0.999$ :

$$
a_{1}(k)=-0.0085 k^{3}+0.1578 k^{2}-0.0024 k+1.9112 .
$$

Accordingly, by combining Equations (16) and (28), an estimated value of the mean wind speed is given by:

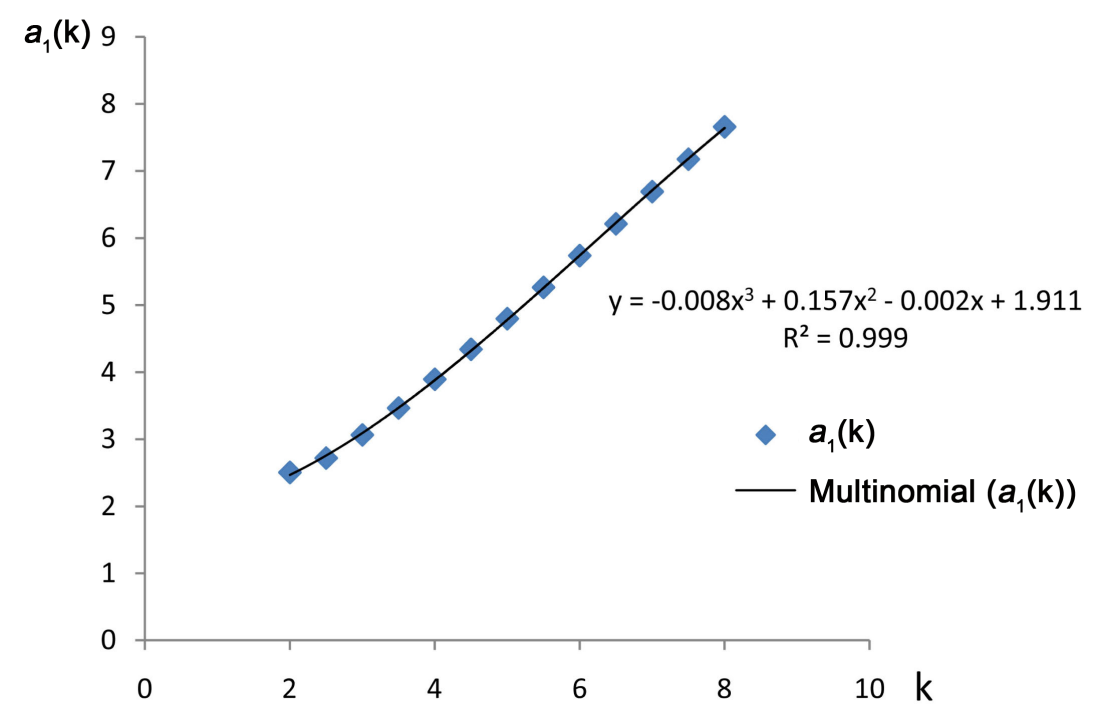

Figure 1. Plotting of $a_{1}(k)$ against $k$. 


$$
\langle v\rangle=c \frac{a_{1}(k)}{k}\left(1-\frac{1}{k}\right)^{1 / k}
$$

The reliability of Equation (30) was tested by using the data of Korhogo mentioned in Section 4.1. The mean wind speeds $\left\langle v_{c}\right\rangle$ calculated by using Equation (30) and the corresponding experimental values $\left\langle v_{e x}\right\rangle$ are displayed in Table 2.

A fairly good agreement is observed between experimental values of $\langle\mathrm{v}\rangle$ and the calculated values gotten by using Equation (30), as the relative errors lie in the range $0.1 \%$ - $4 \%$. It should be noted that the relative errors found for the comparison between the experimental values of $\langle v\rangle$. and the values calculated $g$ by using the relationship of Gash and Twele (Equation (20)), lie in the range $1.5 \%-3.5 \%$, as shown in Table 1 .

Hence, the estimate of the mean speed $\langle v\rangle$ is better by using Equation (30), if compared with the results gotten from the Gash and Twele equation. By using Equation (30), as shown in Table 2, the greatest values of the relative errors are found in July (2.6\%) and December (4.0\%). As a matter of fact, for those two months, the Weibull parameter $k$ is so that $k \geq 7$. For the remaining ten months, where $k<7$, the relative errors lie in the range $0.1 \%-1.6 \%$.

Let $G_{t}$ and $G_{e}$ be respectively the value of $\Gamma(1+1 / k)$ calculated from tables (the exact value) andits estimated value gotten by using Equation (28). A comparison between $G_{t}$ and $G_{e}$ was made. The results of the calculations are displayed in Table 3, where the calculations are made for $k \leq 6$. Table 3 shows a very good agreement between $G_{t}$ and $G_{e}$, since the relative errors lie in the range $0.16 \%-1.5 \%$.

Hence, Equation (28) is a very good estimate of $\Gamma(1+1 / k)$.

Table 1. Comparison between $\left\langle v_{c}\right\rangle(\mathrm{m} / \mathrm{s})$ and $\left\langle v_{e x}\right\rangle(\mathrm{m} / \mathrm{s})$.

\begin{tabular}{ccccccccccccc}
\hline Month & Jan. & Feb. & Mar. & Apr. & May & Jun. & Jul. & Aug. & Sep. & Oct. & Nov. & Dec. \\
\hline$\left\langle v_{e x}\right\rangle$ & 3.42 & 3.34 & 3.22 & 3.50 & 3.52 & 3.21 & 3.37 & 3.06 & 2.68 & 2.66 & 2.58 & 3.18 \\
$\left\langle v_{c}\right\rangle$ & 3.51 & 3.44 & 3.32 & 3.58 & 3.61 & 3.31 & 3.45 & 3.15 & 2.76 & 2.72 & 2.62 & 3.23 \\
Relativeerror (\%) & 2.6 & 3.0 & 3.1 & 2.3 & 2.5 & 2.8 & 2.4 & 2.9 & 3.0 & 2.2 & 1.5 & 1.6 \\
\hline
\end{tabular}

Table 2. Comparison between $\left\langle v_{e x}\right\rangle(\mathrm{m} / \mathrm{s})$ and the calculated mean speed $\left\langle v_{c}\right\rangle(\mathrm{m} / \mathrm{s})$ obtained from Equation (30).

\begin{tabular}{ccccccccccccccc}
\hline Month & Jan. & Feb. & Mar. & Apr. & May & Jun. & Jul. & Aug. & Sep. & Oct. & Nov. & Dec. \\
\hline$\left.v_{e x}\right\rangle$ & 3.42 & 3.34 & 3.22 & 3.50 & 3.52 & 3.21 & 3.37 & 3.06 & 2.68 & 2.66 & 2.58 & 3.18 \\
$\left\langle v_{c}\right\rangle$ & 3.43 & 3.34 & 3.23 & 3.53 & 3.54 & 3.22 & 3.46 & 3.11 & 2.72 & 2.65 & 2.56 & 3.05 \\
Relativeerror (\%) & 1.1 & 0.1 & 0.3 & 0.8 & 0.5 & 0.3 & 2.6 & 1.6 & 1.5 & 0.4 & 0.8 & 4.0 \\
\hline
\end{tabular}

Table 3. Comparison between the exact value and the estimated value of $\Gamma(1+1 / k)$ gotten by using Equation (28).

\begin{tabular}{ccccccc}
\hline$k$ & 2 & 2.5 & 3 & 4.22 & 5.58 \\
\hline$G_{t}$ & 0.8862269 & 0.8872639 & 0.8929796 & 0.9091863 & 0.9239619 \\
$G_{e}$ & 0.8736304 & 0.9007007 & 0.9032822 & 0.9106438 & 0.9337902 & 0.9414530 \\
Relative error (\%) & 1.4 & 1.5 & 1.1 & 0.16 & 1.06 \\
\hline
\end{tabular}




\subsection{2. $\Gamma(1+2 / k)$ Estimate}

Let Equation (15) be considered. The second moment suggests that $\Gamma(1+2 / k)$ may be investigated by using $v^{2} f(v)$. For that purpose, the wind speed for which $v^{2} f(v)$ is maximum was looked for. This wind speed is obtained for

$$
\frac{\mathrm{d}}{\mathrm{d} v}\left[v^{2} f(v)\right]=0 .
$$

The solution of Equation (31) is given by

$$
v=c\left(1+\frac{1}{k}\right)^{1 / k} .
$$

Equations (15) and (32) suggest a link between $\Gamma(1+2 / k)$ and $\left(1+\frac{1}{k}\right)^{1 / k}$.

By introducing a fitting number $a_{2}(k), \Gamma(1+2 / k)$ was expressed by the following relationship:

$$
\Gamma\left(1+\frac{2}{k}\right)=\frac{a_{2}(k)}{k}\left(1+\frac{1}{k}\right)^{1 / k} .
$$

In Equation (33), $a_{2}(k)$ is the fitting number for $n=2 ; b(2)=+1$ is the value of $b(n)$ for $n=1$. For each value of $k, \Gamma(1+2 / k)$ was calculated by using tables. Then $a_{2}(k)$ was calculated from Equation (33). Figure 2 is a plotting of $a_{2}$ (k) against $\mathrm{k}$.

The following polynomial function was found $\left(R^{2}=1\right)$, for $k \leq 6$ :

$$
a_{2}(k)=-0.0053 k^{3}+0.0837 k^{2}+0.502 k+0.3429 .
$$

In order to test the reliability of Equation (33), $G_{t}$ the exact value of $\Gamma(1+2 / k)$ calculated from tables and $G_{e}$, its estimated value gotten by using Equation (33) were compared. The results of the calculations are displayed in Table 4, where the calculations are made for $k \leq 6$.

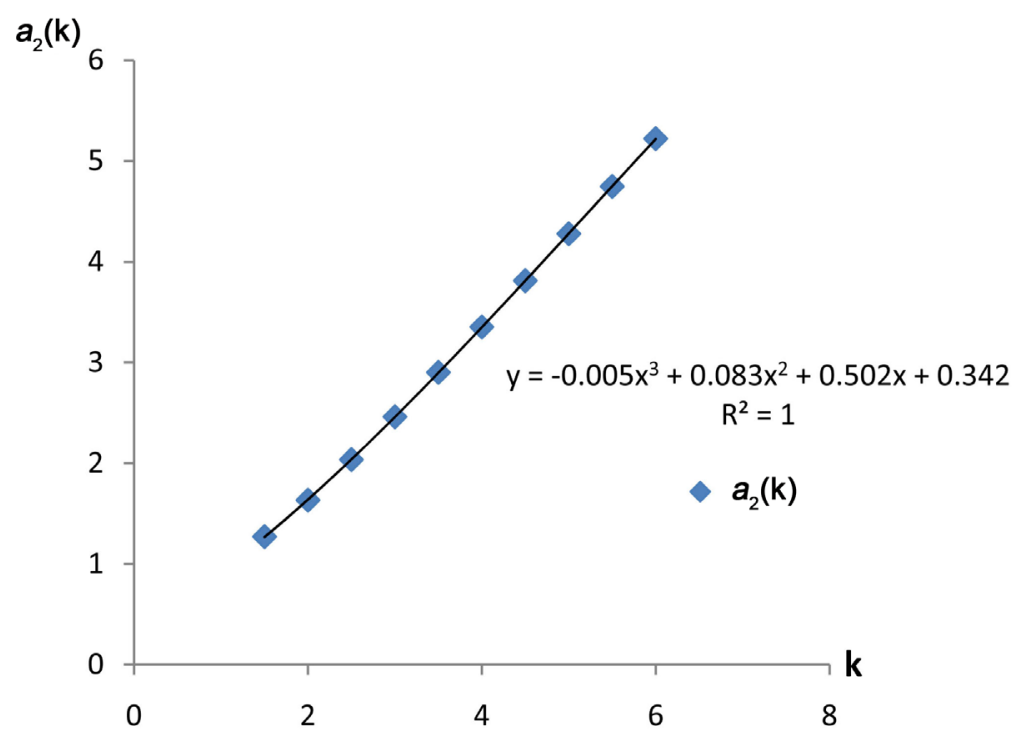

Figure 2. Plotting of $a_{2}(k)$ against $k$. 
Table 4. Comparison between the exact value and the estimated value of $\Gamma(1+2 / k)$ gotten by using Equation (33).

\begin{tabular}{ccccccc}
\hline$k$ & 2 & 3 & 3.5 & 4 & 5 & 6 \\
\hline$G_{t}$ & 1.000000 & 0.9027344 & 0.8905792 & 0.8862269 & 0.8872639 & 0.8929796 \\
$G_{e}$ & 1.0030660 & 0.9025268 & 0.8906764 & 0.8876632 & 0.8923529 & 0.8998236 \\
Relative error (\%) & 0.3 & 0.02 & 0.01 & 0.16 & 0.57 & 0.76 \\
\hline
\end{tabular}

A very good agreement is found between $G_{t}$ and $G_{e}$, since the relative errors, which lie in the range $0.01 \%-0.76 \%$ are all below $1 \%$. It shows that Equation (33) is a very good estimate of $\Gamma(1+2 / k)$.

\subsection{3. $\Gamma(1+3 / k)$ Estimate}

The third moment, obtained from Equation (15), was used to investigate $\Gamma(1+3 / k)$. Hence, the investigation was made by using the product $v^{3} f(v)$. The wind speed for which $v^{3} f(v)$ is maximum was looked for. It is given by the following equation:

$$
\frac{\mathrm{d}}{\mathrm{d} v}\left[v^{3} f(v)\right]=0 .
$$

The following solution of Equation (35) was obtained:

$$
v_{r}=c\left(1+\frac{2}{k}\right)^{1 / k} \text {. }
$$

In the study of the power curve of a wind energy conversion system, $V_{r}$ is deemed to be the rated wind speed [21]. As in the previous cases, Equations (15) and (36) suggest a link between $\Gamma(1+3 / k)$ and $\left(1+\frac{2}{k}\right)^{1 / k}$. Once again, the fitting number $a(k)$ introduced in Equation (25), was used to establish this link, resulting in the following relationship:

$$
\Gamma\left(1+\frac{3}{k}\right)=\frac{a_{3}(k)}{k}\left(1+\frac{2}{k}\right)^{1 / k} .
$$

$a_{3}(k)$ is the fitting number for $n=3 ; b(3)=+2$ is the value of $b$ for $n=3$. The fitting number $a_{3}(k)$ was investigated. For each value of $k, \Gamma(1+3 / k)$ was obtained from tables. Then $a_{3}(k)$ was calculated by using Equation (37). Figure 3 shows a plotting of $a_{3}(k)$ against $k$.

The following polynomial function was found with $R^{2}=0.999$, for $k \leq 6.5$ :

$$
a_{3}(k)=-0.012 k^{3}+0.1958 k^{2}-0.1496 k+1.5179 .
$$

The reliability of Equation (37) was tested. The exact values of $\Gamma(1+3 / k), G_{t}$ calculated from tables and the estimated values $G_{e}$ gotten by using Equation (37) were compared for various values of $k$. The results are displayed in Table 5, where the calculations are made for $2 \leq k \leq 6$. A very good agreement is found between $G_{t}$ and $G_{e}$, since the relative errors lie in the range $0.075 \%-1.47 \%$. 


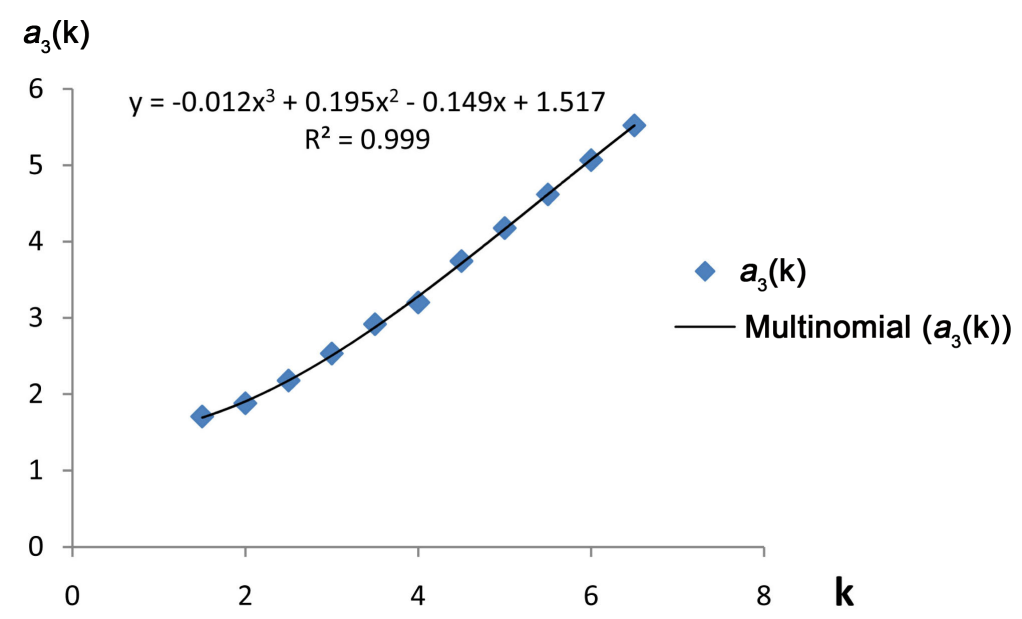

Figure 3. Plotting of $a_{3}(k)$ against $k$.

Table 5. Comparison between the exact value and the estimated value of $\Gamma(1+3 / k)$ obtained by using Equation (37).

\begin{tabular}{ccccccc}
\hline$k$ & 2 & 2.5 & 3 & 4 & 5 & 6 \\
\hline$G_{t}$ & 1.3293403 & 1.1018027 & 1.000000 & 0.9190626 & 0.8935153 & 0.8862269 \\
$G_{e}$ & 1.3456242 & 1.1009763 & 0.9884201 & 0.9055425 & 0.8871348 & 0.8831800 \\
Relative error (\%) & 1.2 & 0.075 & 1.1 & 1.47 & 0.7 & 0.3 \\
\hline
\end{tabular}

Hence, the average wind power density, expressed by Equation (18), can be written as:

$$
\langle P\rangle=\frac{1}{2} \rho c^{3} \frac{a_{3}(k)}{k}\left(1+\frac{2}{k}\right)^{1 / k} .
$$

On the whole, by taking into account Equations (28), (33) and (37), one may write the following equation:

$$
\Gamma\left(1+\frac{n}{k}\right)=\frac{a_{n}(k)}{k}\left(1+\frac{b(n)}{k}\right)^{1 / k} .
$$

In Equation (40), $n$ is an integer number and $b$ is a function of $n$. The corresponding values of $b$ are $b(1)=-1 ; b(2)=+1 ; b(3)=+2$. The fitting number $a_{n}$ $(k)$ is a polynomial function of $k$. It is expressed by Equations (29), (34) and (38) for $n=1, n=2$ and $n=3$ respectively.

A comparative evolution of $a_{1}(k), a_{2}(k)$ and $a_{3}(k)$ with $k$ is given in Figure 4.

An equation giving $b(n)$ as a function of $\mathrm{n}$ was investigated. The following equation was found:

$$
b(n)=-\frac{1}{2} n^{2}+\frac{7}{2} n-4 .
$$

\section{Conclusions}

A practical and simple expression of the gamma function was found, which 


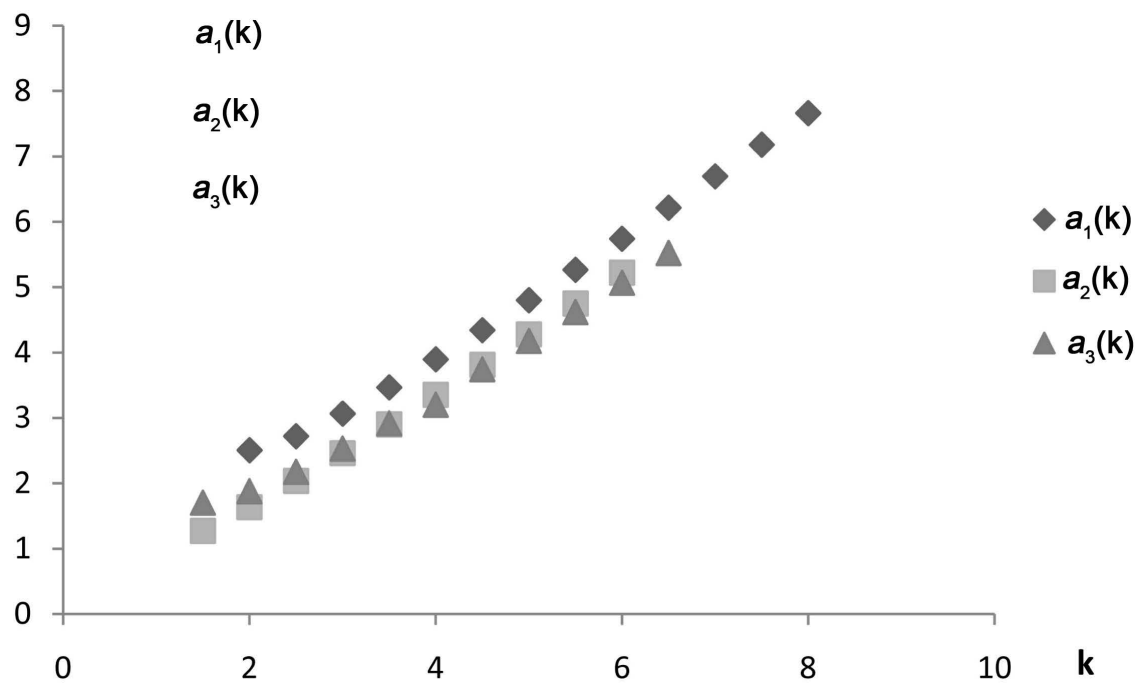

Figure 4. Comparative evolution of $a_{1}(k), a_{2}(k)$ and $a_{3}(k)$ with $k$.

allows the calculation of $\Gamma(1+1 / k), \Gamma(1+2 / k)$ and $\Gamma(1+3 / k)$. In all the three cases, a polynomial fitting function $a_{n}(k)$ was found, with a very high coefficient of determination $R^{2}$. Very good agreements were found between the exact and the estimated values of $\Gamma(1+n / k)$.

The simple expression reported in the paper may be used in wind power theoretical estimate, for the estimation of the mean wind speed $\langle v\rangle$, the variance $\sigma^{2}$ of the wind speed $v$ around the mean speed and the average wind power density. The goodness of the estimate of the gamma function, by using Equation (40), depends on the value of the shape parameter $k$. Very good estimates are gotten for $k \leq 7$. For values of $k$ up to 12 , the estimate remains good, with relative errors not greater than $5 \%$.

\section{Conflicts of Interest}

The author declares no conflicts of interest regarding the publication of this paper.

\section{References}

[1] El-Desouky, B.S., Mustafa, A. and Al-Garash, S. (2017) The Exponential Flexible Weibull Extension Distribution. Open Journal of Modelling and Simulation, 5, 83-97. http://www.scirp.org/journal/ojmsi https://doi.org/10.4236/ojmsi.2017.51007

[2] Weisstein, E.W. (2002) Laplace Transform from Mathworld. A Wolfram Web Resource. http://mathworld.Wolfram.com/LaplaceTransform.html

[3] Godefroy, M. (1901) La fonction gamma, Théorie, Histoire, Bibliographie. Gauthier-Villars, Paris.

[4] Ayant, Y. and Borg, M. (1971) Fonctions spéciales à l'usage des étudiants en physique. Dunod, Paris.

[5] Gourdon, M. and Sabah, P. (1999) Numbers, Constants and Computation. World Wide. http://numbers.computation.free.fr/constants/constants.html 
[6] Sebah, P. and Gourdon, X. (2002) Introduction to the Gamma Function. World Wide. http://numbers.computation.free.fr/constants/constants.html

[7] Boll, M. (1964) Universal Digital Tables for Laboratories and Design Offices. Dunod, Paris.

[8] Abramowitz, M. and Stegun, I. (1964) Handbook of Mathematical Functions. Dover, New York.

[9] Bashuhu, M., Nsabimana, J.C. and Manirakiza, F. (2006) Beta Probability Density Functions and Reference Distributions of Relative Sunshine Duration Data from Burundian Stations. ISESCO Science and Technology Vision, 2, 30-34.

[10] Honerkamp, J. (1999) Stochastic Dynamical Systems: Concepts, Numerical Methods Data Analysis. VCH Publishers, New York, Weinheim, Cambridge.

[11] Ettoumi, F.Y., Mefti, A., Adane, A. and Bouroubi, M.Y. (2002) Statistical Analysis of Solar Measurements in Algeria Using Beta Distributions. Renewable Energy, 26, 47-67. https://doi.org/10.1016/S0960-1481(01)00100-8

[12] Touré, S., Traoré, D. and Traoré, M. (2014) Beta Probability Distribution Function and Relative Wind Speed Distribution. ISESCO Journal of Science and Technology, 10, 26-31.

[13] Weibull, W.A. (1957) Statistical Distribution of Wide Application. Journal of Applied Mechanics, 18, 293-297

[14] Kumar, M. and Samuel, C. (2018) Wind Energy Potential Estimation with Prediction of Wind Speed Distribution. International Journal of Intelligent Systems Technologies Applications, 17, 19-41. https://doi.org/10.1504/IJISTA.2018.091585

[15] Khogall, A., Albar, O.F. and Yoush, B. (1991) Wind and Solar Energy Potential in Makkah (Saudi Arabia). Comparison with Red Sea Costal Sites. Renewable Energy, 1, 453-440. https://doi.org/10.1016/0960-1481(91)90054-S

[16] Feregh, G.M. (1993) Wind Energy Potential in Bahrain. Energy Conversion and Management, 34, 499-506. https://doi.org/10.1016/0196-8904(93)90081-K

[17] Sulaiman, M.Y., Akaah, A.M., Wahab, M.A., Zakaria, A., Sulaiman, Z.A. and Suradi, J. (2002) Wind Characteristic of Oman. Energy, 27, 35-46. https://doi.org/10.1016/S0360-5442(01)00055-X

[18] Wong, R.K.W. (1977) Weibull Distribution, Iterative Likelihood Techniques and Hydrometeorological Data. Journal of Applied Meteorology, 16, 1360-1364. https://doi.org/10.1175/1520-0450(1977)016<1360:WDILTA $>2.0 . C O ; 2$

[19] Touré, S. and Laoualy, R. (2000) A Theoretical Study of Wind Power Potential in Côte D'Ivoire Using the Weibull Distribution. World Renewable Energy Congress VI (WREC), Brighton, UK, 1-7 July 2000, 2304-2307. https://doi.org/10.1016/B978-008043865-8/50498-0

[20] Touré, S. (2005) Investigations on the Eigen-Coordinates Methods for the 2-Parameter Weibull Distribution of Wind Speed. Renewable Energy, 30, 511-521. https://doi.org/10.1016/j.renene.2004.07.007

[21] Sharma, C. and Chadee, J. (2000) Wind as a Reliable Source for Electricity Generation. The Trinidad \& Tobago Perspective. World Renewable Energy Congress VI ( WREC), Brighton, UK, 1-7 July 2000, 1221-1224. https://doi.org/10.1016/B978-008043865-8/50253-1

[22] Gasch, J. and Twele, J. (2002) Wind Power Plants. Fundamentals, Design, Construction and Operation. Solar praxis Berlin James \& James, London. 


\section{Nomenclature}

$B$ : Beta function.

c. the Weibull scale parameter $\left(\mathrm{m} \cdot \mathrm{s}^{-1}\right)$.

$k$ : the Weibull shape parameter.

$p$ : Beta function fitting shape parameter.

$P$ : power density $\left(\mathrm{W} / \mathrm{m}^{2}\right)$.

PDF: Probability Distribution Function.

$q$ : Second beta function fitting shape parameter.

$v$. wind speed $(\mathrm{m} / \mathrm{s})$.

\section{Greek Symbols}

$\Gamma$ : the gamma function.

$\sigma^{2}$ : variance of the wind speed $\left(\mathrm{m}^{2} / \mathrm{s}^{2}\right)$.

$\rho$ : density of air $\left(\mathrm{kg} / \mathrm{m}^{3}\right)$. 